SPECIAL ISSUE

\title{
The AmbuPod Project: Learnings of a Government-Certified, Telemedicine-Enabled, Rural Healthcare Startup in India
}

\author{
Lavanian Dorairaj* MBBS, MD (Aviation Medicine) \\ Cert. Course in Hospital Administration, NIHFW, New Delhi, India; LYNK AmbuPod Pvt Ltd, Bengaluru, India; \\ Former Deputy Director, Medical Services, Indian Air Force, New Delhi, India
}

\section{Abstract}

The Government of India (GoI), during the past 8 years, has been encouraging and supporting start-ups with various schemes, for the purpose of wealth creation, improving economic growth, and employment. There is also a growing support system in India, in the private sector, for funding, mentorship, and techno-commercial support for telemedicine-enabled start-up projects. In practice however, there are many critical decisions that need to be taken and pitfalls to be avoided, for start-ups to achieve success, as envisaged by the GoI.

Objective: This paper shares the challenges encountered so as to help upcoming healthcare start-up's navigate this route skilfully, avoiding pitfalls.

Results: The results of 4 years of work (Jan 2016 to Mar 2020) and the present status are discussed and the learnings drawn from the AmbuPod Project (on-going) summarized.

Conclusion: Telemedicine supported Rural Healthcare Start-Up projects have a good business potential in India, provided challenges are planned for, addressed, and resolved.

Keywords: telemedicine in India; AmbuPod; start-ups in telemedicine; mobile telemedicine clinic

$\mathrm{T}$ elemedicine (TM), a term coined in the 1970s, which literally means 'healing at a distance', signifies the use of information and communications technology to improve patient outcomes by increasing access to care and medical information. The World Health Organization has adopted the following broad description:

The delivery of healthcare services, where distance is a critical factor, by all healthcare professionals using information and communication technologies for the exchange of valid information for diagnosis, treatment and prevention of disease and injuries, research and evaluation and for the continuing education of healthcare providers, all in the interests of advancing the health of individuals and their communities. (1)

Healthcare start-ups in general and those utilizing telemedicine and allied technologies to provide healthcare support in India in particular face major challenges as compared to start-ups in other domains like information technology (IT) and hardware. Some of the challenges encountered are specific to India. The author has been working in rural areas on and off since 1988 and has seen extremely poor health conditions in villages (especially the small and tiny ones) where even basic government assured facilities were not available. In fact, at one health sub-centre, he saw buffaloes resting in the place where healthcare activities were expected to be carried out. In the 1990s during the author's pro bono visits to small and tiny villages in Uttar Pradesh, India, he found that almost $70 \%$ of the illnesses reported could have been resolved by just a nurse or, at most, a general practitioner. The question in his mind was 'why do the rural folks not have access to the same quality of primary healthcare facilities that are assured to the urban population?'. Coming from an armed forces background, he is accustomed to the following two edicts: (a) there must be a primary focus on preventive and primary healthcare to ensure a healthy population and (b) there must be equitable provision of basic healthcare services to all authorized personnel and

*Correspondence: Lavanian Dorairaj. Email: lavanian@gmail.com 
families, irrespective of geographical location. The reasons for this urban-rural disparity are many and prominent amongst them are (a) a large and scattered rural population living in regions with bad or non-existent roads and many having rough terrains (mountains, jungles, deserts, river delta systems), (b) shortage of rural healthcare manpower (2), (c) shortage of equipment and consumables (3), (d) absenteeism and corruption in government healthcare services (4), (e) an extremely low budget for healthcare (India ranks 184th out of 191 in terms of GDP\% spend on healthcare, as per WHO (5)), (f) lack of interest of private entities in providing healthcare services due to the low paying capacity of the rural population (6) and a low level of literacy. The adult literacy rate for females in rural areas is $50.6 \%$ vis-a-vis $76.9 \%$ in urban areas whereas for males the same in rural areas is $74.1 \%$ vis-a-vis $88.3 \%$ in urban areas (7).

\section{Materials and methods}

In spite of the above challenges in rural healthcare, the levels of mobile connectivity in rural India have been improving steadily. For the first time in 2019, rural users (277 million) outnumbered urban areas (227 million) by $10 \%$, thereby eliminating the digital divide that had existed earlier (8). This as per this author, with his two decades of telemedicine expertise, places India in a good position to implement rural telemedicine services. This thought has been shared by many experts and multiple telemedicine projects (government and private) had begun in India as early as the year 2000 (9-12). There, however, was and continues to be, an inherent weakness with telemedicine services. Consultations can be carried out and advice or prescriptions provided to the patient, but how does he get the medicines prescribed and if some lab tests are required, where does he get them done? The patient could be miles away from the nearest lab or pharmacy, and in such a case the whole process of telemedicine would have failed to deliver care to the patient. This conundrum is what the author decided to resolve, with a hybrid rural telemedicine project - the AmbuPod Project, that would deliver a 'circle of care' (described further).

Ideation Phase: The AmbuPod Project originated in 2013-14 while this author was in the Indian state of Uttar Pradesh, implementing a Telemedicine Project around the villages of Hardoi. He was extremely pleased and proud to see semi-literate women - Accredited Social Health Activists (ASHAs) quickly grasping modern technologies like Tablet PCs, software and eLearning, and using them effectively for rural health data capture and for training villagers (many of them illiterate). He was also struck by their eagerness and sincerity while doing this effectively. By 2015, the 'AmbuPod Project' idea had fully taken shape. The main points consolidated were:
1. We can take quality-based healthcare to the villages by training local healthcare resources thereby alleviating manpower shortages.

2. The project would cover the gamut of outpatient primary care and provide these services to each village 6-days-a week.

3. Since patients needed end-to-end care, the AmbuPod Project would be an Hybrid telemedicine project, in which the village patient would interact with a trained person, the locally available AmbuPod manager (APM), who would collect structured data of complaints, history, vitals and other important clinical parameters (including images) thereby adding the human 'touch and feel' element, and upload it on to a cloud-based EMR. A certified allopathic general physician (Tele-Doc) would view the uploaded data and then interact with the patient from his remote office via audio or video conferencing. The APM would be the conduit in all cases and would also translate for the patient, where necessary. The Tele-Doc would then triage the patient and if found fit for telecare, advise and ePrescribe and where necessary, ask for basic clinical/ laboratory tests. The APM would then hand over the medicines to the patient and also carry out the prescribed tests locally. He would also convey the Tele-Doc's advice to the patient thereby completing the circle of care (Fig. 1).

4. Most small villages did not have access to a serviceable ambulance for emergency care and evacuation, so it was decided to add an ambulance (vehicle) to the service offering.

5. The biggest item of cost was the ambulance. It was therefore decided that the team would design a low

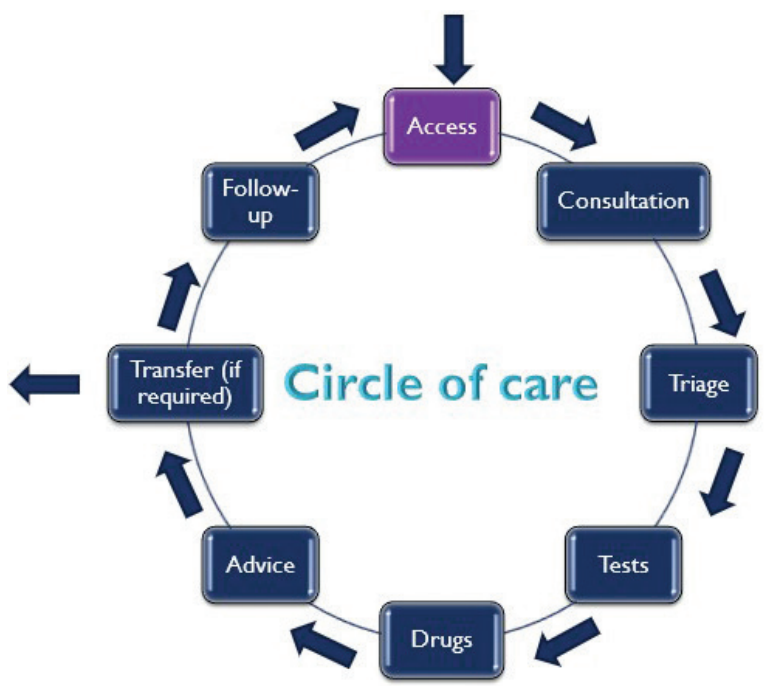

Fig. 1. Completing the circle of healthcare. 
cost, small and narrow-track vehicle, with sufficient space for a lying patient and a sitting assistant, that would be able to run on narrow village roads. This was based on the author's experience that in more than $90 \%$ of cases where an ambulance is called for, only one patient was transferred. This required a unique design (for which a patent was applied for in 2016).

6. This low-cost vehicle, called the AmbuPod ${ }^{\mathrm{TM}}$ was a telemedicine-enabled mobile clinic and one-patient ambulance.

7. A male in rural India earns INR 4,500 on an average (US\$ 61 USD, 51 Euro) per month (13). To ensure affordability for the rural patient each service provided (whether consultation, lab test, ECG or medicines) was planned to be kept below 1USD (0.83 Euro) per patient.

8. Though the fully equipped AmbuPod system was originally designed to cost less than INR 5,00,000 (6,802 USD; 5,638 Euro) an intelligent scheduling protocol was planned so that five to eight villages would be serviced every day thereby bringing down the one-time cost of the AmbuPod by five to eight times, per village.

9. Running costs were planned to be kept low by keeping local infrastructural and AmbuPod manager living costs to a minimum.

\section{Funding phase}

In India it is extremely difficult to get funding partners for healthcare projects because of:

1. High infrastructural and manpower costs

2. Lower level of profits as compared to purely IT/ Hardware/software projects (a large amount of earning in healthcare is the gratefulness of the patient, which is worthless from a hardnosed financial accountant's point of view!). Further, though in 2016 the government's start-up support had just begun, it was extremely cumbersome, red-taped and having long gestation periods.
3. Longer duration to break-even; typically, 3 to 5 years.

4. Lack of 'Star power'. Health care saves lives but does not have the 'pizzazz' or excitement compared to projects involving software/Artificial Intelligence/ biotechnology/gaming hardware.

The author reached out to multiple entities for funding but was informed to return with a viable minimum product (MVP) and then apply for funding.

Since 5,000 under-5 children die every day in India (14) due to lack of healthcare and nutrition the team decided to start immediately and fund the project with whatever funds were available with them/friends/relatives.

\section{Patent design and testing phase}

The design and patent phase till the first prototype took around 24 months. The author applied for an Indian patent No: 201621006069 A on 22 Feb 2016 and the same was published on 25 Aug 2017. A PCT WO/2017/145177 was also applied for the same month. Based on the patent design the first prototype was designed by Jan 2017. This model was a trailer that could be pulled by a motorcycle or any other vehicle (Image 1). It was test run on village roads, dirt tracks and inclines with and without passengers, successfully. Meanwhile, the team registered a private limited company on the 20th of Feb 2017.

The AmbuPod V1 was highly appreciated by doctors, engineers and lay people when it was displayed and demonstrated at Expositions at Pune, Hyderabad and Bengaluru. It was featured on multiple newspapers and 22 TV channels.

Feedback was sought from the drivers, mock patients and doctors regarding the utility, uniqueness, practicality and affordability of the invention. About $82 \%$ appreciated all four parameters; $12 \%$ were critical of one or some of the parameters while $6 \%$ were neutral. Criticisms were mainly related to the size ('too small'), look ('looks like a coffin') and stability of the vehicle motor-cycle combo.

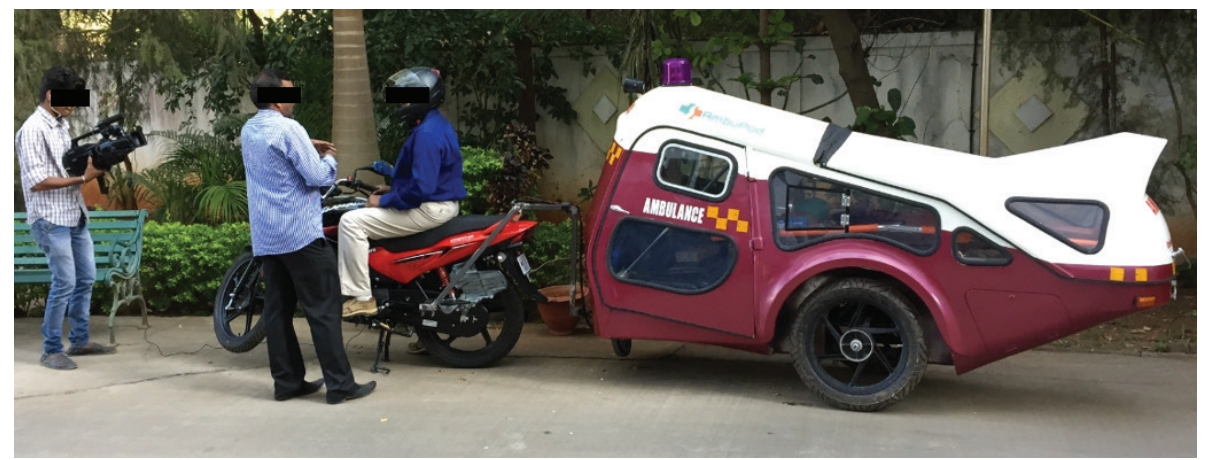

Image 1. A trailer pulled by a motorcycle. 


\section{Certification}

The team faced major difficulties for certifying the product as a new, roadworthy invention. The author first approached the Automotive Research Association of India (ARAI) an autonomous, private organisation affiliated to the Ministry of Heavy Industries and Public Enterprises, Government of India (GoI). A discussion with the Director of the organisation drew a blank as they had no regulations or certification parameters for a trailer ambulance like the AmbuPod. The author was informed that the AmbuPod being a very small motorcycle hauled trailer had no certification parameters yet. The author was advised to reach out to the RTO. The Road Transport Authority (RTA) provides fitness for road use of vehicles in India. They saw the AmbuPod as a trailer and said that they have no rules for certifying it and needed an order from the central government. The team then wrote to the Ministry of Road Transport \& Highways, Government of India for permission to test and run the AmbuPod on public roads. As of date, a reply is still awaited.

Based on the certification issues and feedback from test users, the team moved through three iterations before coming up with the eAmbuPod V2, an electric powered, narrow-track, 3-wheeler based on a certified eRikshaw chassis (Image 2).

The eAmbuPod was well received and we made our first sale to a Chennai-based company in mid-2018 for testing and use in a village near Hyderabad. The eAmbuPod was fully equipped for outpatient services as well as emergency ambulance services. Details of the equipment and software are given in Table 1.

After 1 year of intermittent testing with an 8-month gap (reasons were administrative) the author received feedback that the speed of the eAmbuPod was inadequate (the government had limited the legal speed-limit of eRickshaws to $25 \mathrm{kmph}$ (15.5 miles per hour) only). Other issues were low gradeability, low range and difficulty in charging of the batteries.

Using this feedback the team moved on to version 3 of the AmbuPod that was designed on a certified threewheeler diesel chassis. This would resolve issues related to the speed, gradeability and range. In the first quarter of 2019 , the team again started reaching out to funders for our 100 village pilot. Regrettably, the same funders that had assured us funds on production of an MVP had by now changed their minds. Three of them wanted a viable revenue model running for at least 6 months while the other two wanted a major stake in the company, while

Table 1. The eAmbuPod equipment and software

\begin{tabular}{ll}
\hline Pulse-Oxy meter & Tablet Computer \\
ECG machine & LED Torch \\
Sphygmomanometer & *Electronic LED ENT Scope \\
Stethoscope & ENT Scope \\
Thermometer & Electronic Stethoscope* \\
Glucometer & \#Telemedicine Enabled \\
Covid test* & \#Cloud Apps \\
Hb Strips & \#Live connect and Streaming \\
Ultrasound Doppler Fetoscope & \#EMR \\
Suction Device & IV Drips* \\
Ambu-Bag & Injectables* \\
Oxygen Cylinder and set Nebulizer & Medicines* \\
Light-weight Stretcher & Splints* \\
Weighing machine & Defibrillator* \\
Height measure & ...and more
\end{tabular}

*Additional costs will apply

\#Operational costs shall apply

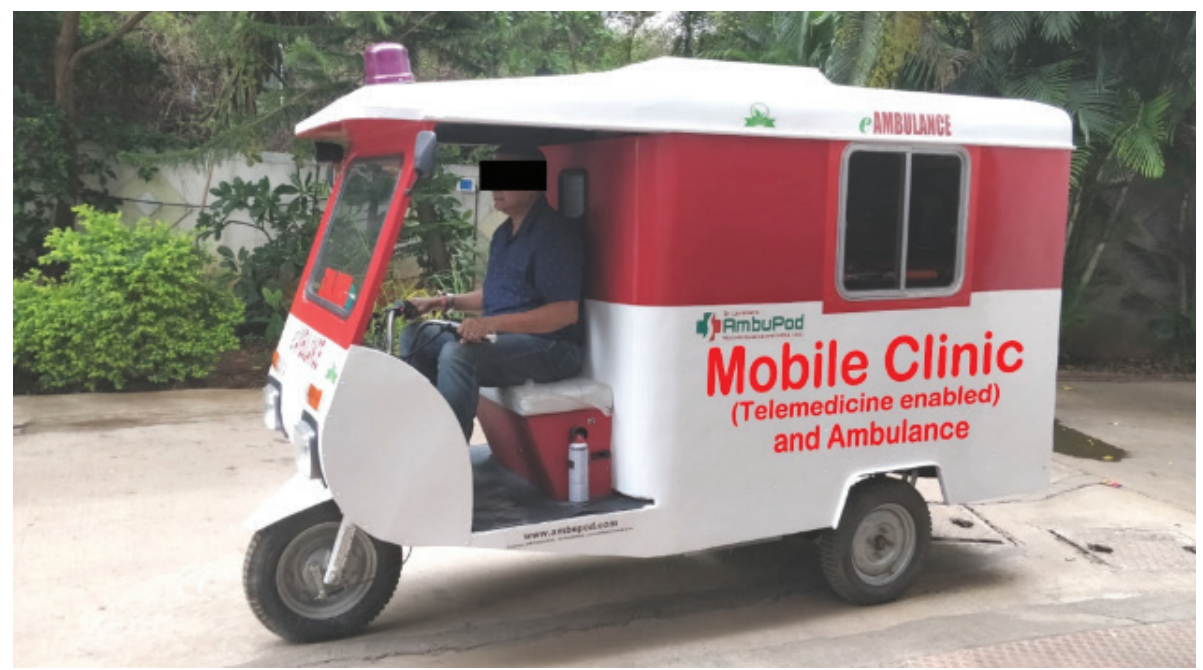

Image 2. A powered electric narrow track 3-wheeler based on a certified eRikshaw chassis. 
promising a very inadequate amount of funds. The team entered contests and won titles, we wrote to multiple NGOs (including the Bill Gates foundation) but no funds came through and we continue to look for partners. Many of the funders felt that we were too ambitious, while some felt that the idea was new and untested, and therefore risky; others said that they could have supported us if we had concentrated on only the Telemedicine software.

By September 2019, we took a major decision to downsize. We shut our corporate office at Pune, removed all staff, moved to a home office at Bengaluru and started a lean finance model where all activities were to be carried out by vendors on a purchase basis only. We had another major administrative activity to carry out, that of moving our registered office from one state to another. This turned out to be a long, arduous and expensive process. By December 2019 we began to get all of our local vendors, processes and business offerings in place and started looking optimistically towards the future.

Unfortunately, in January 2020 the Covid pandemic hit us. All our activities were suspended, while the paperwork lying with the government for moving of our office came to a halt because the governmental office involved in the said activity had shut down.

The team therefore began work-from-home and focused on updating our core telemedicine software so as to become compliant with the Telemedicine Guidelines 2020. These guidelines were released in March 2020 by the Board of Governors, in supersession of the Medical Council of India. Details of our software are given in Table 2.

By October 2020 our team restarted outdoor activities, completed the administrative work and officially moved our company to Bengaluru. These activities were completed by November 2020. Since then, we have re-started our hunt for a funder who would be willing to trust us with around half a million US\$ to become a part owner of a profitable 'Uber of rural healthcare' in India and as a by-product, save thousands of lives and bring a smile on the faces of millions.

\section{Discussion}

Being a doctor from an armed forces background, this sudden jump into entrepreneurship, especially at the age, when many retire, was not easy. If anything, it was only the burning desire to give back to the poorest of the poor of rural India, that kept the author going.

Now most articles and papers discuss success stories, many to the extent of covering/white-washing failures or even deliberately changing data to show success. The author has written this paper with the strong belief that sharing details of issues, problems, failures and challenges and how they were overcome or steered clear of, would go a long way in ensuring that projects by other budding entrepreneurs would have lesser chances of failure and thereby, lesser bankruptcies and more productive output.

\section{Key findings}

So, let us look at the lessons learned.

1. Funding: A project could be based on noble principles or strong emotional connects, however, never design a project with those emotions, use them to buoy you and not dictate to you. Look at any project from the hard, cold, cynical eyes of an accountant with the heart of Shylock (15). After all any project, to succeed, needs money and no funder will provide funds unless he is sure of good (if not massive) returns. The higher the returns, the easier it is to obtain money. It is now easier to obtain seed funds from the government start-up initiatives but be ready to hire a consultant to steer you through the complex process.

Table 2. Details of our software

THE AMBUAPP TELEMEDICINE SOLUTION

\footnotetext{
- Specifically designed for Scenarios III and IV (as per the TPG 2020)

- A cloud based solution with an Android or browser based client (as per choice)

- Works on Android, Windows and Mac

- Works on desktops, laptops, tablets and phones

- The client (spoke) works off-line and on-line

- The hub module is browser based and works in a pure cloud/Hybrid cloud/LAN/WAN configuration

- Built-in EMR

- Focused on structured clinical data capture and analysis

- Online Payments ready
}

- Built-in security powered by the google engine

- Can interoperate with any other HC IT application via HL7 module connects

- ICD I0, SNOMED-CT and LOINC enabled

- ePrescriptions (as per TPG 2020)

- Touch and user friendly

- Directly acquire patient images in one click

- Can capture data from medical devices*

- Plug-and-play ready for primary care

- Modularized and customizable for any speciality(s)

- Low training curve; On line-training

*Device dependent 
2. The Big Idea: Your Big Idea may be all encompassing like 'I wish to take good health to the bottom of the pyramid' but your project should be bite sized, something you can do definitively in a fixed time line say like 'I shall invent a non-invasive blood sugar monitor with an MVP within 9 months'. This could be your first step of your Big Idea of providing good health and it will be infinitely easier to garner support.

3. Patents and designing: The government is now trying to make it easier for start-ups to apply for patients and has fixed rates close to INR 20,000 (374 USD; 227 Euro) per patent, but you will realistically need to keep at least INR 50,000 (684 USD; 567 Euro) for the same. Designing could cost a lot, anywhere from as little as INR 95,000 (1,299 USD; 1,078 Euro; depending on the idea) to several lakhs. The government provides support for designing but their processes are complex, long winding and have fixed dates for applications. Over and above that you may only receive from 40 to $60 \%$ as a grant or loan, the rest has to FIRST come from you. It would also help to join an Incubator. There are now hundreds of these incubators in India and many of them are government sponsored. But be cautious, some of these could be agencies that only provide certain services like an office or patent or legal services which they charge for. If that is all you need, then shop around, you might find something less expensive. Few incubators actually deliver all that they promise to provide. Especially when it comes to mentoring and funding. These aspects are legally covered by words like 'try', 'mostly', 'shall attempt' etc., so if they fail you cannot complain. If you are willing to wait, this is fine; however, if you are in a hurry with tight time lines the author suggests that you first source funding and then get your patent activities carried out through well-known Patent Lawyers.

4. Patent protection: This is a big word that most patent lawyers and the government will use to convince start-ups to apply for patents. It is good to do so; however, do not think that once done nobody can steal your patent....and they will, if it is worth stealing. Even the giants of the world like Samsung, Apple, Google, etc. are fighting patent thieves in international courts. In India, it is extremely difficult to prevent patent theft. This is because of the legal system here where cases can drag on for decades and even if one wins the case, enforcement is weak. To quote an example, our own patent was stolen by the very engineer who designed the AmbuPod for us and in spite of an iron clad agreement and non-disclosure agreement, we could do nothing about it. The answer is to quickly use your patent to saturate the market with your device/idea and become a brand/leader before others have time to copy it. For this you need to be adequately funded.

5. Sources of funding: Funds could be procured from banks or bank like entities. Avoid them if possible until you are making enough profits to be able to pay back the loan. Equity funders would not be interested until you are making good profits and it is better to avoid them till you have a good valuation. So grants and government fundings are the better way to go...unless you have a rich father or uncle or friend who is willing to give you the money (angel funding). Another way is to join an NGO (non-government organisation) that is carrying out activities that are in sync with your Big Idea. To quote an example, say you have an NGO that is monitoring diabetics in rural India and you reach out to them with a proposal for a contactless, reagentless glucose monitor. They might be more inclined to fund you as your invention could save them lots of money. Do remember that no social, government or business entity is truly altruistic, every one of them has their own agenda which ultimately involves money.

6. About partnerships: Never make the mistake of taking up all activities, end to end, of your project. It is better to, as previously mentioned, take only one component to work on. This component should be your core idea. For example, if you wish to run a telemedicine project to detect, treat, monitor and improve the health of diabetics, you need many components like out-reach to the patients, the tests, the doctors providing advice, the telemedicine software, medications, etc. Trying to take up all of these activities could become a major challenge and a cause for failure. Assume that you would like to make the contactless glucometer. It is better to leave the other components to partners while you focus on your core area. Also ensure that the 'big idea' is not discussed with each partner. Most entities work in sealed boxes, they do not know or care to know the big picture. They are completely focused on their activities and it would be wise to share the absolute minimum of information adequate to execute your partnership.

7. Software design: Ensure that your telemedicine software is lean, easy to use, affordable, uses the least resources, has versions in local languages and works effortlessly on bandwidth as low as $2 \mathrm{G}$, since adequate bandwidth and connectivity are a major challenge in rural India. Its ability to work in areas without connectivity would be a major advantage.

\section{Conclusions}

Telemedicine has tremendous potential in India. However, it has yet to attain its full potential due to the many 
factors discussed in this article. This article has described the journey of the author's telemedicine-based rural healthcare project and the challenges faced. It has also suggested solutions so that potential start-ups in this domain can learn from the author's experience, avoid the pitfalls and have a better opportunity to succeed.

\section{References}

1. Author not mentioned. Telemedicine opportunities and developments in member states. World Health Organization; 2010, p. 8. Available from: https://www.who.int/goe/publications/goe telemedicine_2010.pdf [cited 08 January 2021].

2. Sharma DC. India still struggles with rural doctor shortages. Lancet 2015; 386: 2381-2. doi: 10.1016/S0140-6736(15)01231-3

3. Author not mentioned. Rural healthcare sector: a challenge yet to be resolved. Smile Foundation India; 2018. Available from: https://www.smilefoundationindia.org/Media/rural-healthcare. html [cited 08 January 2021].

4. Singh S, Badaya S. Health care in rural India: a lack between need and feed. S Asian J Cancer 2014; 3(2): 143-4. doi: 10.4103/2278-330X.130483

5. Tiwari V. Budget 2020 expectations for India's healthcare sector. Financial Express; 2020. Available from: https://www. financialexpress.com/budget/budget-2020-expectations-for-indias-healthcare-sector/1842519/ [cited 08 January 2021].

6. Barik D, Thorat A. Issues of unequal access to public health in India. Front Public Health 2015; 3: 245. doi: 10.3389/fpubh. 2015.00245

7. Author not mentioned. Women \& Men In India - 2016, Chapter 3- Literacy and education. MOSPI. Available from: http:// mospi.nic.in/sites/default/files/reports_and_publication/
statistical_publication/social_statistics/WM16Chapter3.pdf [cited 08 January 2021].

8. Mitter $\mathrm{S}$. With half a billion active users, Indian internet is more rural, local, mobile-first than ever, Yourstory 13th May 2020. Available from: https://yourstory.com/2020/05/half-billion-active-users-indian-internet-rural-local-mobile-first [cited 08 January 2021].

9. Solberg KE. Telemedicine set to grow in India over the next 5 years. Lancet 2008; 371(9606): 17-18. doi: 10.1016/ S0140-6736(08)60052-5

10. Bagchi S. Telemedicine in Rural India. PLoS Med 2006 Mar; 3(3): e82. doi: 10.1371/journal.pmed.0030082

11. Dasgupta A, Deb S. Telemedicine: a new horizon in public health in India. Indian J Community Med 2008 Jan; 33(1): 3-8. doi: 10.4103/0970-0218.39234

12. Chellaiyan VG, Nirupama AY, Taneja N. Telemedicine in India: where do we stand? J Fam Med Prim Care 2019; 8(6): 1872-6. doi: 10.4103/jfmpc.jfmpc_264_19

13. Statista Research Department. Average earnings from regular employment in rural areas across India from July 2018 to June 2019, by gender(in Indian rupees). Statista Research Department; 2020. Available from https://www.statista.com/statistics/ 1071319/india-average-earnings-of-employee-rural-by-gender [cited 08 January 2021].

14. Ramani K, Mavalankar D, Puwar T, Sanjay J. Why should 5000 children die in India every day? Major causes and managerial challenges. Researchgate; 2010. Available from https://www. researchgate.net/publication/46436824_Why_Should_5000_ Children_Die_in_India_Every_Day_Major_Causes_and_Managerial_Challenges [cited 08 January 2021].

15. Infoplease staff. Merchant of Venice. Infoplease; 2017. Available from: https://www.infoplease.com/primary-sources/books-plays/ tales-from-shakespeare/merchant-venice [cited 08 January 2021]. 